\title{
Estimation of Material Degradation by Backscattered Ultrasonic Tomography
}

\author{
Volodymyr Koshovy, Ihor Romanyshyn, Rostyslav Romanyshyn, Roman Sharamaga \\ Physico-Mechanical Institute of NASU \\ Naukova str. 5, Lviv, Ukraine
}

The problem of the evaluation of operational degradation of material with use of non-destructive methods is actual problem nowadays. The germination and conglomeration of the scattered damages of constructive materials usually occures from the beginning of operation and leads to the changes of material properties, macro-cracks germination and final crack of the product.

The degradation of material is a spatiotemporal process. It can be characterized by localized structure changes in the bulk of the material what leads to the change of spatial distribution of physico-mechanical properties of the material.

In certain papers a solid is considered as an open system in wich the energy comes from outside. On the open systems theory two types of evolutional processes are usually distinguished - degradation and selforganization. The degradation is connected with the order decrease (randomness increase) of the system. Its estimation can be carried out on the base of statistical approaches alowing the estimation of system degree of the order (randomness).

In material science the degradation of the materials is considered as the process of germination and conglomeration of scattered damages of the material. It occures from the beginning of operation and leads to the changes of the material properties, the germination of macrocracks and final crack of the product. Microscopy investigations show that the fracture kinetics of polycrystalline materials can be divided into three stages:

- $\quad$ the formation of scattered over the bulk of material germinal micropores and microcracks (with an average size 0,1-3 microns);

- $\quad$ association of micropores and microcracks to a critical length wich is defined by the size of structural heterogeneity (10-100 microns);

- abrupt localization of plastic deformation, formation and growth of the macrodefect, avalanche destruction of the product.

Three-stage kinetics of changes in metal properties (ultrasonic velocity, Young's modulus, electrical resistance) is usually pointed in fatigue tests.

It should be noted that the three-stage way (successive periods of evolution of the system: "burnishing", "normal" mode and "aging") is often used in the theory of reliability (whyle the analysys of the rate of machine failures while the time of operating), demographical investigations, seismic investigations.

The phenomenological approaches - direct and undirect - are used for damage evaluation. The direct methods include fractography. The indirect methods consist in the measurment of deviation of the physico-mechanical properties such as dencity, elasticy modulus, microhardness, and electrical resistance.

One of the first works in witch the connection between products reliability and probablistic imaginations is represented is the statistical model of the distruction of ideally brittle product in the place of it's weakest element (Aleksandrov A. P., Zhurkov S. N., 1933). This paper represents the experimental material about the dissperce of strength limits for the thin glass fibers of different diameters and different lengths, and describes the scale effect of the strength limit of the product average reduce while their's size increase. This effect is based on the locality of brittle destruction.

Weibull's distribution is widely used for the description of a lot of processes. It is based on the following. The product is considered as a connection of elementary volumes. It will be destroyed only in case of the destruction of one of the volumes. That means that durability of the brittle product is defined by the durability of it's weakest element. Weibull destribution is 2-parameter. One of it's parameters characterize the homogeneity of the destribution. It can be used in the reliability theory for the heterogeneity of the material evaluation (more parameter, the material more homogeneous).

The statistic theory of the damage accumulation is developed too. In the most fundamental work in this direction is the one in which it is shown that the process of material fragmentation can be described by lognormal (ie exponential) distribution of particle sizes. Two conditions must be sytisfied: different parts of the product are fragmented independently; sizes of fragments are proportional to the time of process. Probably at the initial stage of fracture this conditions are satisfied while the process of the microcracks 
(of much more smaller lengths that the granule size) appearance. The lognormal distribution is used while the examination of the granule size in solid.

The function of microcracks length distribution change is mentioned before the appearance of the localized crack. This fact indicates about a transition from one hierarchical damage degree to another as a result of previous defects merging. Such a change of distribution functions is usually observed while dynamic or cyclic loadind.

One of the approaches of the damage degree of the constructive materials estimation is based on the repeated measurment of the hardness (or other physico-mechanical property of the material) and calculation of the statistical charecteristics of the measured values, for example hardness dispersal parameters. The physical reasoning of this approach is the fact that the degree of physico-mechanical characteristics dispersal of the material depends on its structural conditions usually changing while material degradation.

Howewer this approach doesn't allow us to get the degradation degree estimation in the bulk of the product, for example by depth, although it is well known that the degradation degree, for example in the pipelines, usually reduces by depth while operating.

The methodology of degradation degree estimation is based on the following assumptions.

1. It is well known that material degradation is accompanied by the localized amplification of structural heterogeneities. It leads to the increase of physico-mechanical properties of material dispersion.

2. The scattered ultrasonic signal is one of the most sencitive to the structure change parameters.

3. Tomographical methos are one of the most informative and noiseproof diagnostic approaches. The informativity of tomographical approaches consists in the fact that they allow to get the information about spatial distribution of the material properties in the bulk of product on base of product sounding from surface. The noise immunity of tomographical methods is caused by the fact that high-frequency filtering is beeing performed while tomographical reconstruction by overlaying a set of projections. So tomographical methods allow to get information about material properties even in case this information cant be received from sigle measurments because of noises.

4. The degradation of material degree evaluation consists in the estimation of randomness of tomographical images if scattering ability in material.

A method of ultrasonic tomography on base of back scattered signal is developed. The method allows to reconstruct the spatial distribution of scattering ability in the bulk of cylindric products and consists in the following.

1. Ultrasonic scanning of the product from outer surface; back-scattered signal registration in the form of A-scan; A-scan processing; spheric projection forming

2. C-scan building. C-scan is the image representing scattering ability averaged over the thickness of the product (1). With the help of C-scan the anomalous regions are identified to be investigated with the methods of 3D-tomography.

$$
S(i, j)=\sum_{k=1}^{N} \frac{S_{k}{ }^{2}}{N},
$$

$s_{k}(k=1, \ldots, N)$ - values of samples;

$N$ - number of samples.

3. 3D-tomographical reconstruction of the spatial distribution of scattering ability.

The qauantitive estimation of the randomness of spatial distribution can be calculated on the base of Signal-to-Noise Ratio for the reconstructed tomographical images (2).

$$
S N R=10 \lg \frac{\sum_{i} \sum_{j} a_{i j}^{2}}{\sum_{i} \sum_{j}\left(a_{i j}-\bar{a}\right)^{2}},
$$

$a_{i j}(i=1,2, \ldots, N ; j=1,2, \ldots M)-$ pixels;

$\bar{a}=\frac{1}{M N} \sum_{i=1}^{N} \sum_{j=1}^{M} a_{i j}$ - average.

In case of homogeneous image: $a_{i j}=\bar{a}(i=1,2, \ldots, N ; j=1,2, \ldots M) S N R \rightarrow \infty$. 
The degradation is accompanied by the increase of randomness degree of spatial distribution. That means that SNR reduces.

One should note that the positive feature of the ratio is that it doesn't depend on the value of $a_{i j}$ only on the randomness of distribution.

SNR can be calculated over whole controlled object, over tomographical images or over arbitary curves (depending on operating conditions or controll methods).

Lower and upper parts of horizontal pipeline usually run in different conditions. Calculating of SNR of radial sections can allow estimation of degradation degree.

It is well known that the inner part of the pipeline has a higher degradation degree while its operating. In this case one should calculate SNR of equidistant from the axis of the pipeline lines. This eliminates the methodological error inherent in ultrasound imaging on the scattered signal and associated with the signal attenuation. Reduce of the degradation from inner surface to outer surface will be accompanied by the increase of SNR. This trend will not take place for the pipelines without operating time, and the parameter SNR is greater than for pipelines with operating time.

\section{Experimental approbation of assessment of operating degradation methodology}

Consider a thick-walled cylindrical product (Fig. 1), by the surface of which scanning by direct ultrasonic transducer with the step $\Delta s$ and registration of the back scattered by the structure of the material ultrasonic signal in the form of A-Scan in the control points are carried out.

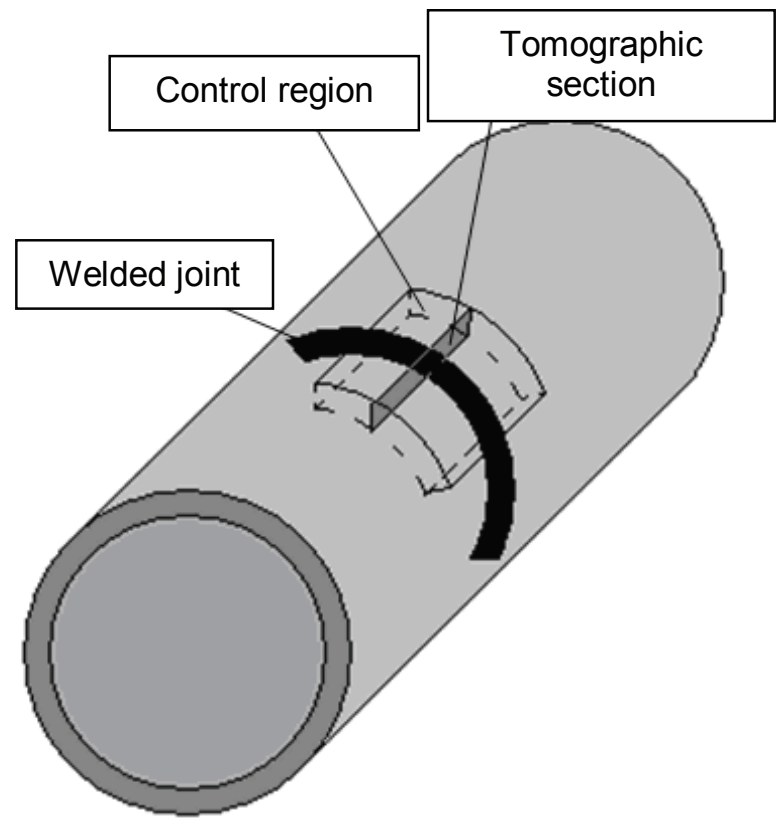

Fig. 1. Geometry of data collection and tomographic reconstruction

Evaluation of material degradation by the thickness of the cylindrical product was conducted by calculating the SNR on the base of tomographic image of ultrasound scattering material ability in the individual cross sections of cylindrical product along the equidistant from the axis of the cylinder lines.

The calculating of SNR along the equidistant from the axis of the cylinder lines, eliminates the error which is typical for ultrasound tomography on back scattered signal and is connected with signal attenuation.

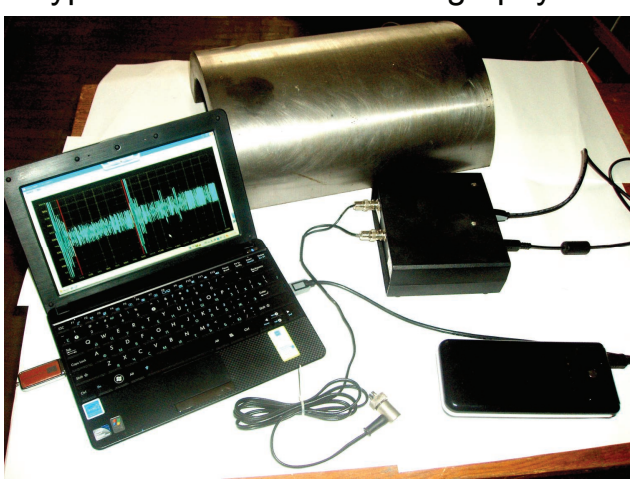
The degradation of metal pipeline) will change by thickness (from inner to outer surface) and will be accompanied by changes in value of SNR. For the pipeline without operating time (without degradation) this trend should not take place, and the signal-noise ratio should be higher than for pipelines with operating time.

A computerized ultrasound tomograph based on registration of back scattered signal for degree of degradation by the thickness of metal pipe estimation was developed (Fig. 2).

The results of experimental testing of the methods of 
assessing the degree of operational degradation of the metal by thickness in the pipeline for three objects of control:

- feed water pipeline of one of the second circuit blocks in Rivne nuclear power plant (industrial conditions), which has $\sim 250000$ hours of operating time;

- a new pipeline segment, given by the Rivne nuclear power plant, a metal which has no operating time;

- the segment of pipeline with unknown operating time from TPP are presented

Three individual tomographic sections and SNR for pipelines with different operating time are given on Fig. 3.

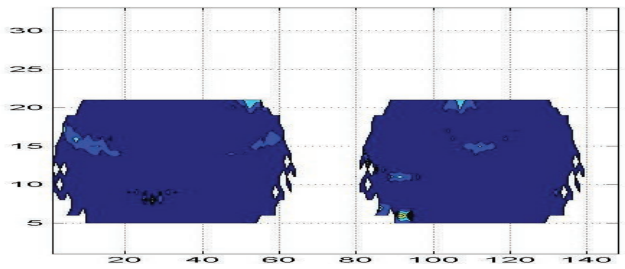

a)
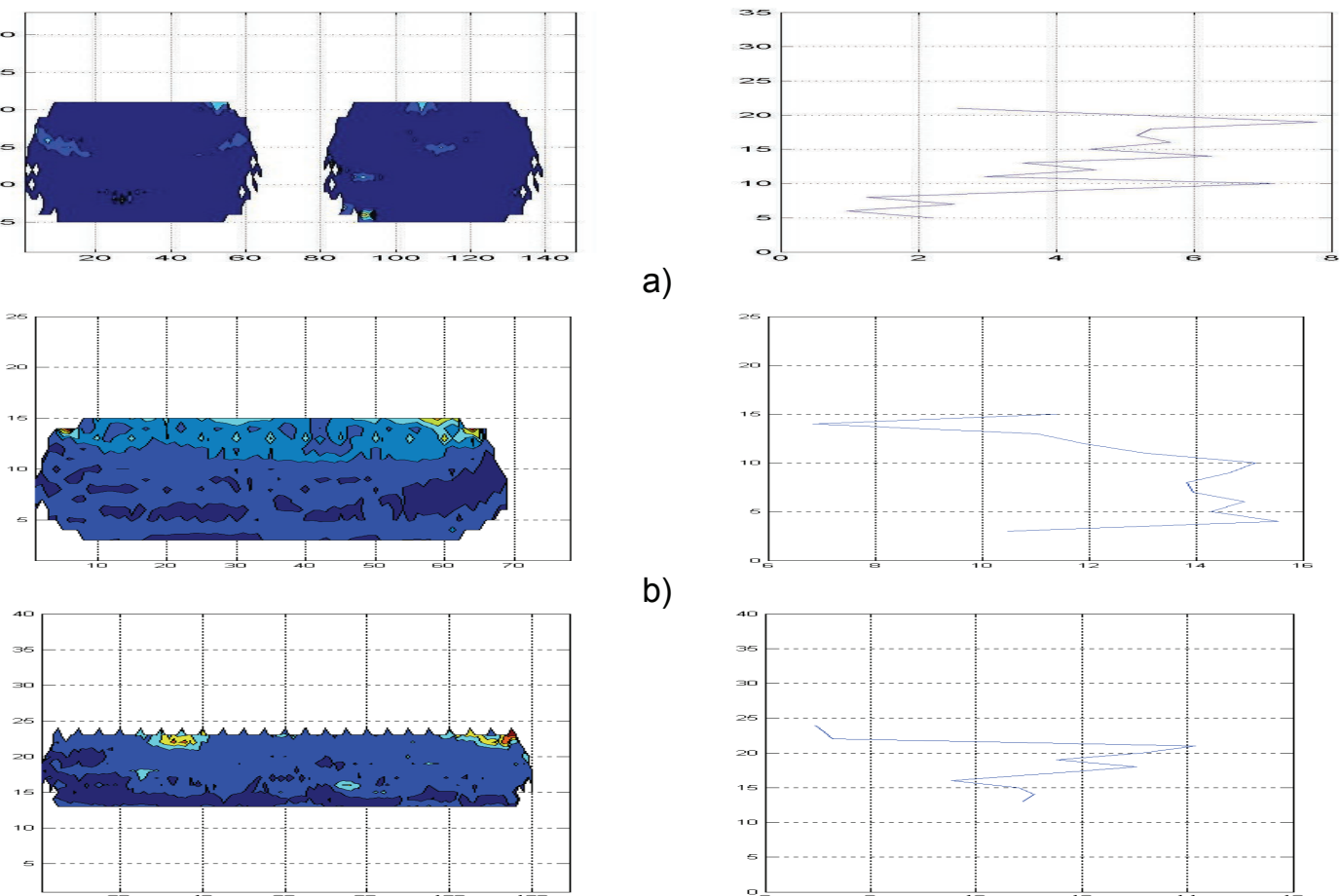

b)

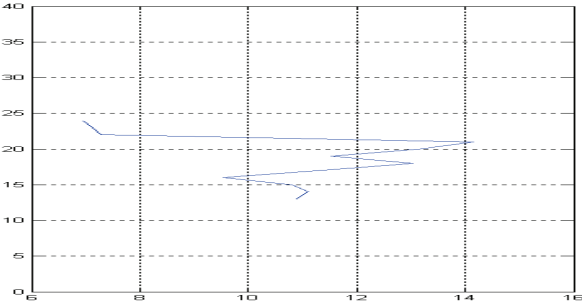

c)

Fig. 3. Tomographic image sections and SNR by the thickness of the pipeline (a - 250000 hours of operating time, $\mathrm{b}$ - without operating time, $\mathrm{c}-$ pipeline from TPP with unknown opearating time)

As it is demonstrated by experimental researches SNR value is within $2 \div 7$ and changes from internal to external surface of the pipeline for the object with operating time $\sim 250000$ hours that reflects a change of degradation of metal degree. For an object without operating time the value of SNR is in the range $14 \div$ 15 and there are no significant change by its thickness.

A Nondestructive method of experimental evaluation of operating time (OT) of metal pipeline on the basis of experimental determination of SNR is proposed (Fig. 4).

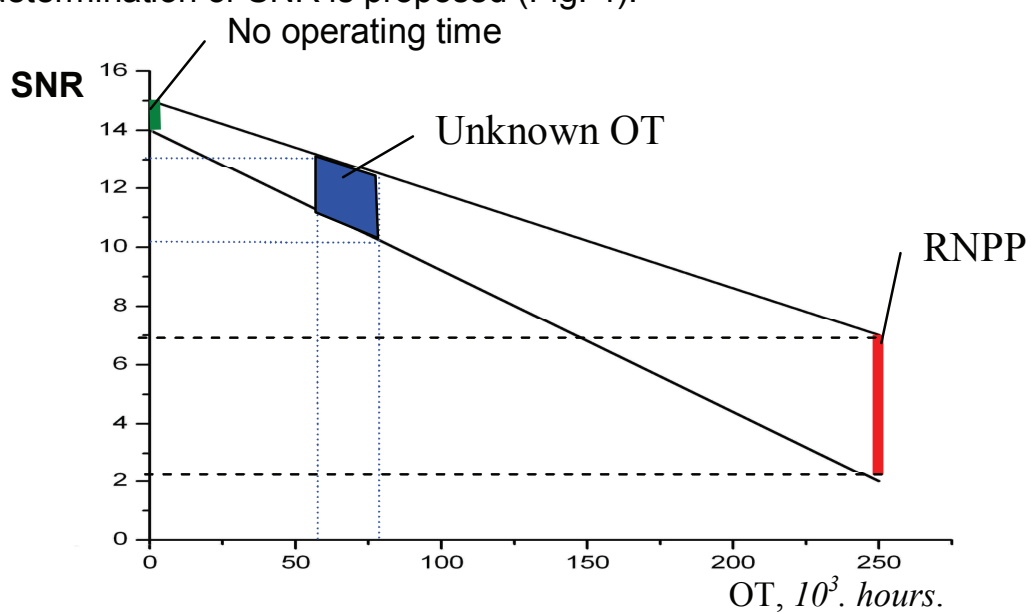

Fig. 4. Method of determination operating time of the pipeline based on SNR 